\title{
Project YES! Youth engaging for success: A peer mentoring program to transition youth to HIV self-management in Zambia
}

Project SOAR

Follow this and additional works at: https://knowledgecommons.popcouncil.org/departments_sbsr-hiv

Part of the Demography, Population, and Ecology Commons, Family, Life Course, and Society Commons, International Public Health Commons, and the Medicine and Health Commons How does access to this work benefit you? Let us know!

\section{Recommended Citation}

Project SOAR. 2017. "Project YES! Youth engaging for success: A peer mentoring program to transition youth to HIV self-management in Zambia," Activity brief. Washington, DC: Population Council. 


\section{Project YES! Youth Engaging for Success: A peer mentoring program to transition youth to HIV self-management in Zambia}

As adolescents and young adults living with HIV age into adulthood, they must navigate the transition to HIV self-management and adult care. This is a complex process, particularly for young people who must manage a chronic illness like HIV. As a result, many adolescents and young adults living with HIV have incomplete adherence to antiretroviral therapy (ART), leading to poor viral suppression and a greater risk of HIV-related mortality. As of December 2016, preliminary analysis of routine viral load (VL) testing conducted among HIV-positive, adolescent clinic attendees at the Arthur Davison Children's Hospital (ADCH) in Ndola, Zambia revealed that approximately 50 percent have VL failure $(\geq 1,000$ copies $/ \mathrm{ml}$ ), underscoring the urgent need for new strategies to assist this population in adhering to care and treatment. Unfortunately, there is little data from the region on how to support youth in having a greater and more effective role in the management of their own care.

This operational study responds to these challenges and evidence gaps by refining and testing a peermentoring strategy in Zambia to strengthen the capacity of health systems and families to support

Research Partners: Johns Hopkins Bloomberg School of Public Health (JHSPH) and Arthur Davison Children's Hospital (ADCH), Ndola, Zambia

Location: Ndola, Zambia

Study Duration: 2016-2019

For more information, contact Dr. Julie

Denison, JHSPH, jdenison@jhu.edu

or Dr. Jonathan K. Mwansa, ADCH, jonathankmwansa@gmail.com.

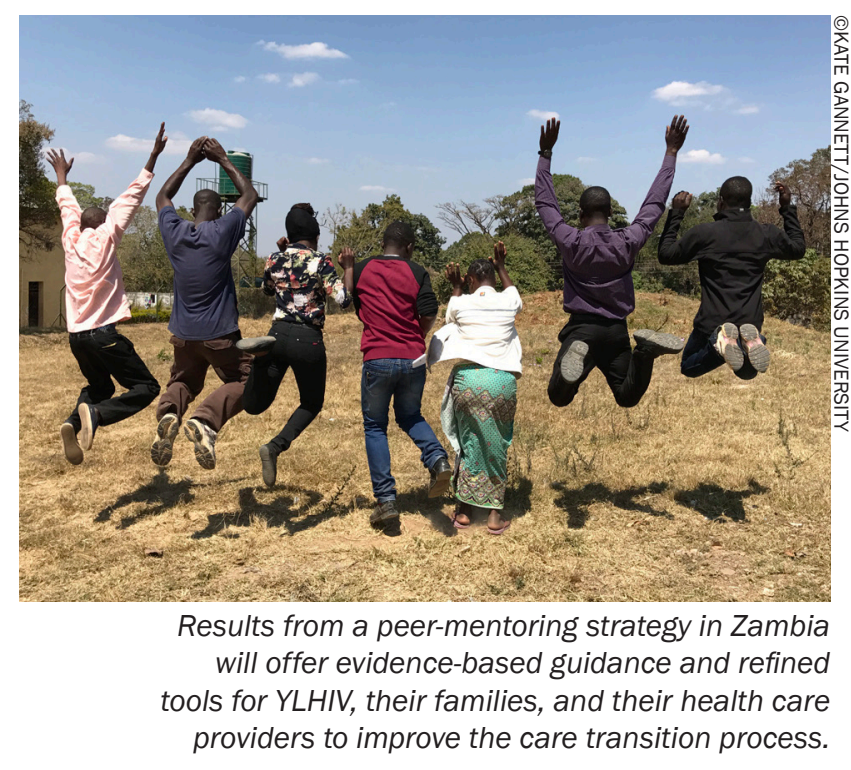

youth as they transition to, and engage in, selfmanagement and adult HIV care and treatment. This approach builds on previous Ministry of Healthapproved research at ADCH with youth living with HIV (YLHIV) and their families. Results will offer evidence-based guidance and refined tools for YLHIV, their families, and their health care providers to improve the care transition process and related HIV outcomes.

\section{OUR RESEARCH}

The study is a randomized controlled trial (RCT) at the individual level that uses a stepped wedge design to assess a peer-mentoring strategy and toolkit to support youth living with HIV (ages 15-24 years) achieve a successful transition to selfmanagement and adult care. The study team will test a six-month peer-mentoring program among 144 youth in an intervention arm compared to 144 youth in a comparison arm. RCT participants will be systematically selected from two hospitals-ADCH and Ndola Teaching Hospital (NTH)-and two primary care clinics-Twapia Clinic and Lubuto Clinic. 
After the completion of the initial 6-month intervention and the subsequent 6-month follow-up assessment among participants in both arms, the primary intervention group will enter a maintenance phase and the comparison group will receive the intervention for six months. Assessments, including a survey and a blood draw for VL testing, will occur at baseline, at the end of the first six months, and at the end of 12 months. Resistance testing will also be conducted at baseline for those participants who have VL failure.

At the end of the initial six-month intervention, we will conduct qualitative in-depth interviews with participants who were randomized to the intervention arm, their caregivers when possible, and the health care providers and peer mentors involved in the program. This data will provide contextual details and experiences to help interpret the study findings and determine what aspects of the intervention were valued by participants.

\section{THE INTERVENTION}

Peer mentors will be HIV-positive young adults who have successfully transitioned to self-management. These peer mentors will undergo an initial twoweek pre-service training to prepare them to be skilled, valued, and paid members of the health care system. The initial pre-service training will be complemented by on-going in-service training on key topics selected by the peer mentors and project staff. Interactions with the youth participants will take the form of one-on-one and youth group meetings. These interactions will be guided by tools in the Toolkit for Transition of Care and Other Services for Adolescents Living with HIV, developed by AIDSTAR-One. During initial one-on-one meetings, the peer mentors and youth participants will work together to develop a customized action plan to prioritize topics for the meetings based on the needs of the participant. Because a key goal of the project is VL suppression, each meeting will also include check-in questions on adherence.

\section{What do we mean by transitioning?} Transition of care is a multifaceted, active process that must address the medical, psychosocial, and educational or vocational needs of adolescents as they move from a childfocused to an adult-focused health-care system.

- For some youth living with HIV, the process includes a physical transition from a pediatric or adolescent model of care to an adult facility.

- Many youth living with HIV in sub-Saharan Africa already receive care in adult HIV clinics yet still need support to develop the skills to self-manage HIV.

\section{RESEARCH UTILIZATION}

The findings from this study will directly benefit clinic services for youth living with HIV by testing an intervention designed to improve HIV treatment outcomes as they transition into adult care and/ or HIV self-management. Specific mechanisms for ensuring research utilization include: (a) engaging key stakeholders, such as Ministry of Health $(\mathrm{MOH})$ officials (including technical working groups) and community HIV groups, through meetings, office visits, and study updates throughout the research; (b) holding a data interpretation workshop to present preliminary findings and facilitate discussion of their implications with clinic staff and other key stakeholders; and (c) holding a dissemination meeting during which participants, consisting of public health officials and clinic staff (including youth peer mentors), community groups, and youth living with HIV and their caregivers, will have a chance to review the data and actively guide next steps and recommendations. The study team will share the data with the $\mathrm{MOH}$ and the study sites, and discuss ways of sustaining the intervention post-study if deemed effective.
Project SOAR/Population Council

4301 Connecticut Avenue, NW, Suite 280

Washington, DC 20008

Tel: +12022379400

e-mail: ProjectSOAR@popcouncil.org

popcouncil.org/ProjectSOAR

(c)Population Council, August 2017 